\title{
Jacobi-Perron Algorithms, Bi-Orthogonal Polynomials and Inverse Scattering Problems
}

\author{
By \\ Yoshifumi KATO* and Kazuhiko Аомото**
}

\section{§ 0. Introduction}

It is well-known that the spectral theory of a Jacobi matrix is essentially equivalent to the theory of orthogonal polynomials with respect to its spectral density on a real interval. This is also related to the Padé approximation of a Stieltjes integral by rational functions which gives us the inverse spectral theory of Jacobi matrices (See [19] and [20]).

On the other hand several people have shown that the equations of Toda lattices are described by Lax type formalism of Jacobi matrices and related to its spectral densities (See [2], [6], [7], [8] and [10]).

It seems to be interesting to ask whether these facts can be generalized to higher order linear difference operators. The purpose of this note is to construct 3rd order linear difference operators by JacobiPerron algorithms as a generalization of continued fractions (See [5]) and to prove an equivalence between linear evolution equations of spectral densities and Lax type equations (See Theorem 1 and 2).

\section{§1. Jacobi-Perron Algorithms}

Let two Radon measures $\mu_{0}(d \zeta), \mu_{1}(d \zeta)$ be given on a compact set $\Gamma$ in $C$. We consider the Stieltjes integrals

Communicated by S. Hitotumatu, June 1, 1983.

* Department of Engineering Mathematics, Faculty of Engineering, Nagoya University, Nagoya 464, Japan.

** Department of Mathematics, Faculty of Science, Nagoya University, Nagoya 464, Japan. 


$$
\omega_{j}(z)=\int_{\Gamma} \frac{\mu_{j}(d \zeta)}{z-\zeta}, \quad 0 \leqq j \leqq 1,
$$

which are holomorphic with respect to $z \in \boldsymbol{C}-\Gamma$. Then the functions $\omega_{j}(z)$ have Laurent expansions at the infinity as follows:

$$
\omega_{j}(z)=\sum_{\nu=0}^{\infty} c_{j, \nu} z^{-\nu-1}
$$

where $c_{j, \nu}$ denote the moments:

$$
c_{j, \nu}=\int_{\Gamma} \zeta^{\nu} \mu_{j}(d \zeta) .
$$

We assume the following "regularity condition" holds for $\mu_{0}(d \zeta)$ and $\mu_{1}(d \zeta):$

( $\mathscr{H} .1)$ All the determinants of order $2 k$ and $2 k+1$ for the sequence $\left\{c_{0, \nu}, c_{1, \nu}\right\}_{0 \leqq \nu<\infty}$

$$
\begin{aligned}
& \operatorname{det}\left[\begin{array}{l}
\left(c_{0, i+j}\right)_{0 \leqq i \leqq k-1,0 \leqq j \leqq 2 k-1} \\
\left(c_{1, i+j}\right)_{0 \leqq i \leqq k-1,0 \leqq j \leqq 2 k-1}
\end{array}\right], \quad k \geqq 1, \\
& \operatorname{det}\left[\begin{array}{l}
\left(c_{0, i+j}\right)_{0 \leqq i \leqq k, 0 \leqq j \leqq 2 k} \\
\left(c_{1, i+j}\right)_{0 \leqq i \leqq k-1,0 \leqq j \leqq 2 k}
\end{array}\right], \quad k \geqq 0,
\end{aligned}
$$

are different from zero for $k \geqq 0$. These will be denoted by $D_{2 k}$ and $D_{2 k+1}$ respectively. We put $D_{0}$ to be equal to 1 .

We can put

$$
\omega_{0}=\frac{u_{1}}{w_{1}}, \omega_{1}=\frac{v_{1}}{w_{1}},
$$

such that $u_{1}, v_{1}, w_{1}$ are described as Laurent series as follows:

$$
\left\{\begin{array}{l}
u_{1}=1, \\
v_{1}=\xi_{0}+\frac{\xi_{1}}{z}+\frac{\xi_{2}}{z^{2}}+\cdots, \\
w_{1}=\eta_{-1} z+\eta_{0}+\frac{\eta_{1}}{z}+\frac{\eta_{2}}{z^{2}}+\cdots,
\end{array}\right.
$$

where $\eta_{-1}=1 / c_{0,0}$ and $\xi_{0}=c_{1,0} / c_{0,0}$ are well-defined. We apply the Jacobi-Perron algorithm of 3rd order (see [5]) to (1.7) at the infinity $z=\infty$ in the following manner:

$$
\left\{\begin{array}{l}
\frac{v_{1}}{u_{1}}=\alpha_{2}+\frac{u_{2}}{w_{2}}, \\
\frac{w_{1}}{u_{1}}=\beta_{2} z+\beta_{2}^{\prime}+\frac{v_{2}}{w_{2}},
\end{array}\right.
$$


where $u_{2} / w_{2}=O\left(z^{-1}\right)$ and $v_{2} / w_{2}=O\left(z^{-1}\right)$. Namely we consider a projective transformation

$$
\left[\begin{array}{l}
u_{1} \\
v_{1} \\
w_{1}
\end{array}\right]=\left[\begin{array}{lll}
0 & 0 & 1 \\
1 & 0 & \alpha_{2} \\
0 & 1 & \beta_{2} z+\beta_{2}^{\prime}
\end{array}\right]\left[\begin{array}{l}
u_{2} \\
v_{2} \\
w_{2}
\end{array}\right],
$$

between the points $u_{1}: v_{1}: w_{1}$ and $u_{2}: v_{2}: w_{2}$ in $\boldsymbol{C} P^{2} \cdot u_{2} / w_{2}$ and $v_{2} / w_{2}$ are also expressed by Laurent series :

$$
\left\{\begin{array}{l}
\frac{u_{2}}{w_{2}}=\sum_{\nu=0}^{\infty} \frac{c_{0 . \nu}^{\prime}}{z^{\nu+1}} \\
\frac{v_{2}}{w_{2}}=\sum_{\nu=0}^{\infty} \frac{c_{1, \nu}^{\prime}}{z^{\nu+1}} .
\end{array}\right.
$$

Then we can define the similar determinants $D_{2 k}^{\prime}$ and $D_{2 k+1}^{\prime}$ as $D_{2 k}$ and $D_{2 k+1}$ respectively for the sequence $\left\{c_{0, \nu}^{\prime}, c_{1, \nu}^{\prime}\right\}_{0 \leqq \nu<\infty}$. First we show

\section{Lemma 1.}

$D_{2 k}^{\prime}=c_{0,0}^{-3 k-1} D_{2 k+1}$,

$D_{2 k+1}^{\prime}=(-1)^{k} c_{0,0}^{-3 k-2} D_{2 k+2}$,

for $k \geqq 0$.

Proof. The relation (1.8) implies the following identities:

$$
\begin{aligned}
& \alpha_{2} c_{0, n}+\sum_{\nu=0}^{n-1} c_{0, \nu}^{\prime} c_{0, n-1-\nu}=c_{1, n}, \quad n \geqq 0, \\
& \beta_{2} c_{0, n}+\beta_{2}^{\prime} c_{0, n-1}+\sum_{\nu=0}^{n-2} c_{1, \nu}^{\prime} c_{0, n-\nu}=0, n \geqq 0 .
\end{aligned}
$$

Then we have firstly

$$
a_{2}^{\prime}=\frac{c_{1,0}}{c_{0,0}}, \beta_{2}=\frac{1}{c_{0,0}}, \beta_{2}^{\prime}=\frac{-c_{0,1}}{c_{0,0}^{2}} .
$$

Successive uses of the relations $(1.12) \sim(1.14)$ give the formula

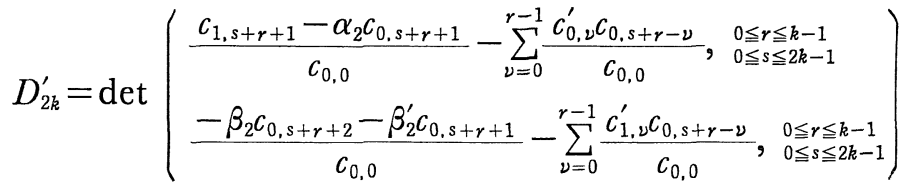

$$
\begin{aligned}
& =\frac{1}{c_{0,0}} \operatorname{det}\left(\begin{array}{c|ccc}
c_{0,0} & c_{0,1} c_{0,2} & c_{0,2 k} \\
\hline c_{0, k-1}^{\prime} & \frac{c_{1, s+r+1}-\alpha_{2} c_{0, s+r+1}}{c_{0,0}}-\sum_{\nu=0}^{r-2} \frac{c_{0, \nu}^{\prime} c_{0, s+r-\nu}}{c_{0,0}}, & 0 \leqq r \leqq k-1 \\
0 \leqq s \leqq 2 k-1 \\
\\
c_{1, k-1}^{\prime} & \frac{-\beta_{2} c_{0, s+r+2}-\beta_{2}^{\prime} c_{0, s+r+1}}{c_{0,0}}-\sum_{\nu=0}^{r-2} \frac{c_{1, \nu}^{\prime} c_{0, s+r-\nu}}{c_{0,0}}, \begin{array}{c}
0 \leqq r \leqq k-1 \\
0 \leqq s \leqq 2 k-1
\end{array}
\end{array}\right),
\end{aligned}
$$


for $c_{0,-1}^{\prime}=0$, which is equal to $c_{0,0}^{-3 k-1} D_{2 k+1}$. We have thus proved $(1.11)_{2 k}$. (1.11) $)_{2 k+1}$ can be proved similarly.

Since $D_{2 k}$ and $D_{2 k+1}, k \geqq 0$, are all different from zero, $D_{2 k}^{\prime}$ and $D_{2 k+1}^{\prime}, k \geqq 0$, are also different from zero. In particular we have $c_{0,0}^{\prime} \neq 0$. This makes it possible to repeat the substitution (1.8) for $\left(v_{2} / u_{2}, w_{2} / u_{2}\right)$ instead of $\left(v_{1} / u_{1}, w_{1} / u_{1}\right)$ and so on. Therefore the regularity property allows us to consider successively the $n$-th JacobiPerron transform

$$
\left[\begin{array}{l}
u_{n} \\
v_{n} \\
w_{n}
\end{array}\right]=\left[\begin{array}{lll}
0 & 0 & 1 \\
1 & 0 & \alpha_{n+1} \\
0 & 1 & \beta_{n+1} z+\beta_{n+1}^{\prime}
\end{array}\right]\left[\begin{array}{c}
u_{n+1} \\
v_{n+1} \\
w_{n+1}
\end{array}\right],
$$

for $n \geqq 2$. By the same reason, we can also see all the coefficients $\beta_{n}=D_{n+1} / D_{n}, n \geqq 2$, are different from zero. If we take $\omega_{0}=v_{0} / u_{0}$, $\omega_{1}=w_{0} / u_{0}$, then $u_{0}, v_{0}, w_{0}$ are linearly related to $u_{n}, v_{n}, w_{n}$ as follows :

$$
\left[\begin{array}{c}
u_{0} \\
v_{0} \\
w_{0}
\end{array}\right]=\left[\begin{array}{ccc}
p_{n}(z) & p_{n+1}(z) & p_{n+2}(z) \\
p_{n}^{\prime}(z) & p_{n+1}^{\prime}(z) & p_{n+2}^{\prime}(z) \\
p_{n}^{\prime \prime}(z) & p_{n+1}^{\prime \prime}(z) & p_{n+2}^{\prime \prime}(z)
\end{array}\right]\left[\begin{array}{c}
u_{n} \\
v_{n} \\
w_{n}
\end{array}\right], \quad n \geqq 3,
$$

where $p_{n}(z), p_{n}^{\prime}(z), p_{n}^{\prime \prime}(z)$ denote polynomials of exact degree $n-3$, $n-4, n-4$ respectively. The matrix in the right hand side will be abbreviated by $A_{n}(z)$. Note that $\operatorname{det} A_{n}(z)=1$, so that $A_{n}(z)$ and ${ }^{t} A_{n}(z)^{-1}$ lie in $G L_{3}(\boldsymbol{C}[z])$. Therefore ${ }^{t} A_{n}(z)^{-1}$ can be written

$$
{ }^{t} A_{n}(z)^{-1}=\left[\begin{array}{ccc}
\tilde{p}_{n}(z) & \tilde{q}_{n}(z) & \tilde{r}_{n}(z) \\
\tilde{p}_{n}^{\prime}(z) & \tilde{q}_{n}^{\prime}(z) & \tilde{r}_{n}^{\prime}(z) \\
\tilde{p}_{n}^{\prime \prime}(z) & \tilde{q}_{n}^{\prime \prime}(z) & \tilde{r}_{n}^{\prime \prime}(z)
\end{array}\right],
$$

where $\tilde{p}_{n}, \tilde{q}_{n}, \tilde{r}_{n}, \tilde{p}_{n}^{\prime}, \tilde{q}_{n}^{\prime}, \tilde{r}_{n}^{\prime}, \tilde{p}_{n}^{\prime \prime}, \tilde{q}_{n}^{\prime \prime}, \tilde{r}_{n}^{\prime \prime}$ denote polynomials. This algorithm to compute sequences of polynomials $p_{n}(z), p_{n}^{\prime}(z), p_{n}^{\prime \prime}(z)$ and $\tilde{p}_{n}(z)$, $\tilde{p}_{n}^{\prime}(z), \tilde{p}_{n}^{\prime \prime}(z)$ is nothing else than the usual Jacobi-Perron algorithm with respect to Laurent series.

Proposition 2. (i) Each polynomial $\varphi_{n}=p_{n}, p_{n}^{\prime}$, $p_{n}^{\prime \prime}$ satisfies the difference equation:

$$
\varphi_{n+3}=\varphi_{n}+\alpha_{n+1} \varphi_{n+1}+\left(\beta_{n+1} z+\beta_{n+1}^{\prime}\right) \varphi_{n+2}, \quad n \geqq 1,
$$

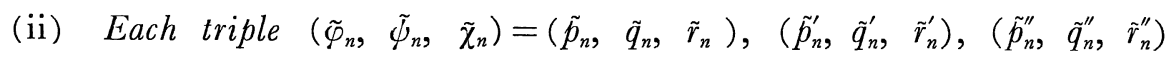


satisfies the difference equations

$$
\left\{\begin{array}{l}
\tilde{\varphi}_{n+1}=-\alpha_{n+1} \tilde{\varphi}_{n}+\tilde{\psi}_{n}, \\
\tilde{\varphi}_{n+1}=-\left(\beta_{n+1} z+\beta_{n+1}^{\prime}\right) \tilde{\varphi}_{n}+\tilde{\chi}_{n}, \\
\tilde{\chi}_{n+1}=\tilde{\varphi}_{n} .
\end{array}\right.
$$

Proof. In fact these follow from the obvious recurrence equation for $A_{n}(z)$ :

$$
A_{n+1}(z)=A_{n}(z)\left[\begin{array}{lll}
0 & 0 & 1 \\
1 & 0 & \alpha_{n+1} \\
0 & 1 & \beta_{n+1} z+\beta_{n+1}^{\prime}
\end{array}\right] .
$$

Since all $\beta_{n}$ are different from zero, we can see from (1.20) that the degrees of $\tilde{p}_{n}, \tilde{q}_{n}, \tilde{r}_{n}, \tilde{p}_{n}^{\prime}, \tilde{q}_{n}^{\prime}, \tilde{r}_{n}^{\prime}, \tilde{p}_{n}^{\prime \prime}, \tilde{q}_{n}^{\prime \prime}, \tilde{r}_{n}^{\prime \prime}$ are exactly equal to $k-2$, $k-1, k-2, k-1, k, k-1, k-1, k-1, k-2$ if $n=2 k$ and equal to $k-1, k, k-1, k, k, k-1, k-1, k, k-1$ if $n=2 k+1$ respectively.

Proposition 3. ( i) For a pair of measures $\mu_{0}(d \zeta), \mu_{1}(d \zeta)$ on $\Gamma$ which have the regularity property ( $\mathscr{H} .1)$, the Jacobi-Perron algorithm can be carried out up to any stage. We have the following Padé approximation for $\omega_{0}(z), \omega_{1}(z)$ at the infinity $z=\infty$ :

$$
\begin{aligned}
& \left\{\begin{array}{l}
\omega_{0}-p_{2 k}^{\prime} / p_{2 k}=O\left(z^{-3 k+3}\right), \\
\omega_{1}-p_{2 k}^{\prime \prime} / p_{2 k}=O\left(z^{-3 k+4}\right), \quad k \geqq 1,
\end{array}\right. \\
& \left\{\begin{array}{l}
\omega_{0}-p_{2 k+1}^{\prime} / p_{2 k+1}=O\left(z^{-3 k+2}\right), \\
\omega_{1}-p_{2 k+1}^{\prime \prime} / p_{2 k+1}=O\left(z^{-3 k+2}\right), \quad k \geqq 1 .
\end{array}\right.
\end{aligned}
$$

(ii) The function

$$
u_{n} / u_{0}=\tilde{p}_{n}+\tilde{p}_{n}^{\prime} \omega_{0}+\tilde{p}_{n}^{\prime \prime} \omega_{1}, \quad n \geqq 1,
$$

has the property

$$
u_{n} / u_{0}=O\left(z^{-n}\right)
$$

Proof. First we want to prove (1.24). Since $u_{n+1}=-\alpha_{n+1} u_{n}+v_{n}$ and $v_{n} / u_{n}=\alpha_{n+1}+O\left(z^{-1}\right)$, we have

$$
\frac{u_{n+1}}{u_{n}}=O\left(z^{-1}\right)
$$


This shows (1.25) by induction on $n$. Next we want to prove the first part of (1.22). The left hand side is equal to

$$
\begin{aligned}
\frac{v_{0}}{u_{0}}-\frac{p_{2 k}^{\prime}}{p_{2 k}} & =\frac{p_{2 k}^{\prime} u_{2 k}+p_{2 k+1}^{\prime} v_{2 k}+p_{2 k+2}^{\prime} w_{2 k}}{p_{2 k} u_{2 k}+p_{2 k+1} v_{2 k}+p_{2 k+2} w_{2 k}}-\frac{p_{2 k}^{\prime}}{p_{2 k}} \\
& =\frac{\left(p_{2 k} p_{2 k+1}^{\prime}-p_{2 k}^{\prime} p_{2 k+1}\right) v_{2 k}+\left(p_{2 k} p_{2 k+2}^{\prime}-p_{2 k}^{\prime} p_{2 k+2}\right) w_{2 k}}{u_{0} p_{2 k}} \\
& =\frac{\tilde{r}_{2 k}^{\prime \prime} v_{2 k}-\tilde{q}_{2 k}^{\prime \prime} w_{2 k}}{u_{0} p_{2 k}} \\
& =\frac{u_{2 k}\left(\tilde{r}_{2 k}^{\prime \prime} \frac{v_{2 k}}{u_{2 k}}-\tilde{q}_{2 k}^{\prime \prime} \frac{w w_{2 k}}{u_{2 k}}\right)}{u_{0} p_{2 k}} .
\end{aligned}
$$

On the other hand, we have that $u_{2 k} / u_{0}=O\left(z^{-2 k}\right), v_{2 k} / u_{2 k}=O(1)$, $w_{2 k} / u_{2 k}=O(z), \operatorname{deg} p_{2 k}=2 k-3, \operatorname{deg} \tilde{r}_{2 k}^{\prime \prime}=k-2, \operatorname{deg} \tilde{q}_{2 k}^{\prime \prime}=k-1$. Thus,

$$
\frac{v_{0}}{u_{0}}-\frac{p_{2 k}^{\prime}}{p_{2 k}}=O\left(z^{-3 k+3}\right) \text {. }
$$

For the second part we have

$$
\begin{aligned}
\frac{w_{0}}{u_{0}}-\frac{p_{2 k}^{\prime \prime}}{p_{2 k}} & =\frac{-\tilde{r}_{2 k}^{\prime} v_{2 k}+\tilde{q}_{2 k}^{\prime} w_{2 k}}{u_{0} p_{2 k}} \\
& =O\left(z^{-3 k+4}\right) .
\end{aligned}
$$

(1.23) can be proved similarly.

Conversely, under the condition $(\mathscr{H} .1)$ the triples $\left(p_{n}, p_{n}^{\prime}, p_{n}^{\prime \prime}\right)$ or $\left(\tilde{p}_{n}, \tilde{p}_{n}^{\prime}, \tilde{p}_{n}^{\prime \prime}\right)$ are the unique ones except for constant factors such that they satisfy (1.22), (1.23) or (1.25) among polynomials of the same degrees as $\left(p_{n}, p_{n}^{\prime}, p_{n}^{\prime \prime}\right)$ or $\left(\tilde{p}_{n}, \tilde{p}_{n}^{\prime}, \tilde{p}_{n}^{\prime \prime}\right)$. Namely the triples $\left(p_{n}, p_{n}^{\prime}, p_{n}^{\prime \prime}\right)$ or $\left(\tilde{p}_{n}, \tilde{p}_{n}^{\prime}, \tilde{p}_{n}^{\prime \prime}\right)$ are "perfect systems" in the sense of $\mathrm{K}$. Mahler (See [13] and [14]). In fact (1.22) and (1.23) imply

$$
\begin{aligned}
& \left\{\begin{array}{l}
\omega_{0} p_{2 k}-p_{2 k}^{\prime}=O\left(z^{-k}\right), \\
\omega_{1} p_{2 k}-p_{2 k}^{\prime \prime}=O\left(z^{-k+1}\right),
\end{array}\right. \\
& \left\{\begin{array}{l}
\omega_{0} p_{2 k+1}-p_{2 k+1}^{\prime}=O\left(z^{-k}\right), \\
\omega_{1} p_{2 k+1}-p_{2 k+1}^{\prime \prime}=O\left(z^{-k}\right),
\end{array}\right.
\end{aligned}
$$

which give linear equations with respect to the coefficients of $p_{2 k}, p_{2 k}^{\prime}$, $p_{2 k}^{\prime \prime}$ or $p_{2 k+1}, p_{2 k+1}^{\prime}, p_{2 k+1}^{\prime \prime}$. The determinants coincide with (1.4) and (1.5). Owing to $(\mathscr{H} .1),(1.22)^{\prime}$ and $(1.23)^{\prime}$ determine uniquely the polynomials $p_{2 k}(z)$ and $p_{2 k+1}(z)$ except for constant factors. These give us the following formulae: 


$$
p_{2 k}(z)=\mid \begin{array}{cccc}
c_{0,0} & c_{0,1} & \cdots & c_{0,2 k-3} \\
c_{0,1} & c_{0,2} & \cdots & c_{0,2 k-2} \\
\vdots & \vdots & \cdots & \vdots \\
c_{0, k-2} & c_{0, k-1} & \cdots & c_{0,3 k-5} \\
c_{1,0} & c_{1,1} & \cdots & c_{1,2 k-3} \\
\vdots & \vdots & & \vdots \\
c_{1, k-3} & c_{1, k-2} & \cdots & c_{1,3 k-6} \\
1 & z & \cdots & z^{2 k-3} \\
p_{2 k+1}(z)=\left|\begin{array}{cccc}
c_{c, 0} & c_{0,1} & \cdots & c_{0,2 k-2} \\
c_{0,1} & c_{0,2} & \cdots & c_{0,2 k-1} \\
\vdots & \vdots & & \vdots \\
c_{0, k-2} & c_{0, k-1} & \cdots & c_{0,3 k-4} \\
c_{1,0} & c_{1,1} & \cdots & c_{1,2 k-2} \\
\vdots & \vdots & \cdots & \vdots \\
c_{1, k-2} & c_{1, k-1} & \cdots & c_{1,3 k-4} \\
1 & z & \cdots & z^{2 k-2}
\end{array}\right|
\end{array}
$$

These are rewritten in integral form by using the relation (1.3) as follows :

$$
\begin{aligned}
& p_{2 k}(z)=\sum_{\substack{i_{1}<\cdots<i_{k-1} \\
j_{1}<\cdots<j_{k-2} \\
\left({ }_{1} \cdots, \cdots, i_{k-1}\right) \amalg\left(j_{1}, \cdots, j_{k-2}\right)=(1,2, \cdots, 2 k-3)}} \int_{\Gamma}\left(z-z_{1}\right)\left(z-z_{2}\right) \cdots\left(z-z_{2 k-3}\right) \\
& \text { 。 } \prod_{1 \leqq i<j \leqq 2 k-3}\left(z_{j}-z_{i}\right) \cdot \prod_{1 \leqq \alpha<\beta \leqq k-1}\left(z_{i_{\beta}}-z_{i_{\alpha}}\right) \cdot \prod_{1 \leqq \gamma<\delta \leqq k-2}\left(z_{j_{\delta}}-z_{j_{\gamma}}\right) \\
& \cdot \mu_{0}\left(d z_{i_{1}}\right) \cdots \mu_{0}\left(d z_{i_{k-1}}\right) \cdot \mu_{1}\left(d z_{j_{1}}\right) \cdots \mu_{1}\left(d z_{j_{k-2}}\right) \text {, } \\
& \begin{array}{c}
p_{2 k+1}(z)=\sum_{\substack{i_{1}<\cdots<i_{k-1} \\
j_{1}<\cdots<j_{k-1} \\
\left(i_{1}, \cdots, i_{k-1}\right) \amalg\left(j_{1}, \cdots, j_{k-1}\right)=(1, \cdots, 2 k-2)}} \int_{\Gamma}\left(z-z_{1}\right)\left(z-z_{2}\right) \cdots\left(z-z_{2 k-2}\right) \\
\left(z_{1}, z_{i}\right)
\end{array} \\
& \cdot \prod_{1 \leqq i<j \leqq 2 k-2}\left(z_{j}-z_{i}\right) \cdot \prod_{1 \leqq \alpha<\beta \leqq k-1}\left(z_{i_{\beta}}-z_{i_{\alpha}}\right) \cdot \prod_{1 \leqq \gamma<\delta \leqq k-1}\left(z_{j_{\delta}}-z_{j_{\gamma}}\right) \\
& \cdot \mu_{0}\left(d z_{i_{1}}\right) \cdots \mu_{0}\left(d z_{i_{k-1}}\right) \mu_{1}\left(d z_{j_{1}}\right) \cdots \mu_{1}\left(d z_{j_{k-1}}\right) \text {. }
\end{aligned}
$$

Owing to (1.1), the left hand side of $(1.22)^{\prime}$ is equal to

$$
\begin{aligned}
& \int_{\Gamma} \frac{p_{2 k}(z) \mu_{0}(d \zeta)}{z-\zeta}-p_{2 k}^{\prime}(z) \\
& =\int_{\Gamma} \underbrace{\frac{\left(p_{2 k}(z)-p_{2 k}(\zeta)\right) \mu_{0}(d \zeta)}{z-}-p_{2 k}^{\prime}(z)}_{\text {polynomial part }}+\int_{\Gamma} \frac{p_{2 k}(\zeta) \mu_{0}(d \zeta)}{z-\zeta} .
\end{aligned}
$$


Therefore we have

$$
\begin{aligned}
& p_{2 k}^{\prime}(z)=\int_{\Gamma} \frac{p_{2 k}(z)-p_{2 k}(\zeta)}{z-\zeta} \mu_{0}(d \zeta), \\
& O\left(z^{-k}\right)=\int_{\Gamma} \frac{p_{2 k}(\zeta) \mu_{0}(d \zeta)}{z-\zeta} .
\end{aligned}
$$

Similarly

$$
\begin{aligned}
& p_{2 k}^{\prime \prime}(z)=\int_{\Gamma} \frac{p_{2 k}(z)-p_{2 k}(\zeta)}{z-\zeta} \mu_{1}(d \zeta), \\
& O\left(z^{-k+1}\right)=\int_{\Gamma} \frac{p_{2 k}(\zeta) \mu_{1}(d \zeta)}{z-\zeta},
\end{aligned}
$$

$$
p_{2 k+1}^{\prime}(z)=\int_{\Gamma} \frac{p_{2 k+1}(z)-p_{2 k+1}(\zeta)}{z-\zeta} \mu_{0}(d \zeta),
$$

$$
O\left(z^{-k}\right)=\int_{\Gamma} \frac{p_{2 k+1}(\zeta) \mu_{0}(d \zeta)}{z-\zeta}
$$

$$
p_{2 k+1}^{\prime \prime}(z)=\int_{\Gamma} \frac{p_{2 k+1}(z)-p_{2 k+1}(\zeta)}{z-\zeta} \mu_{1}(d \zeta)
$$

$$
O\left(z^{-k}\right)=\int_{\Gamma} \frac{p_{2 k+1}(\zeta) \mu_{1}(d \zeta)}{z-\zeta} .
$$

In particular from (1.36), (1.38), (1.40) and (1.42) the following bi-orthogonality holds:

$$
\begin{array}{ll}
\int_{\Gamma} \zeta^{n} p_{2 k}(\zeta) \mu_{0}(d \zeta)=0, & 0 \leqq n \leqq k-2, \\
\int_{\Gamma} \zeta^{n} p_{2 k}(\zeta) \mu_{1}(d \zeta)=0, & 0 \leqq n \leqq k-3, \\
\int_{\Gamma} \zeta^{n} p_{2 k+1}(\zeta) \mu_{0}(d \zeta)=0, & 0 \leqq n \leqq k-2, \\
\int_{\Gamma} \zeta^{n} p_{2 k+1}(\zeta) \mu_{1}(d \zeta)=0, & 0 \leqq n \leqq k-2 .
\end{array}
$$

(1.19) can be rewritten as follows :

$$
z \varphi_{n+2}=-\frac{\varphi_{n}}{\beta_{n+1}}-\frac{\alpha_{n+1}}{\beta_{n+1}} \varphi_{n+1}-\frac{\beta_{n+1}^{\prime}}{\beta_{n+1}} \varphi_{n+2}+\frac{\varphi_{n+3}}{\beta_{n+1}} .
$$

Namely the operators of multiplication by $z$ gives a matrix representation as follows : 


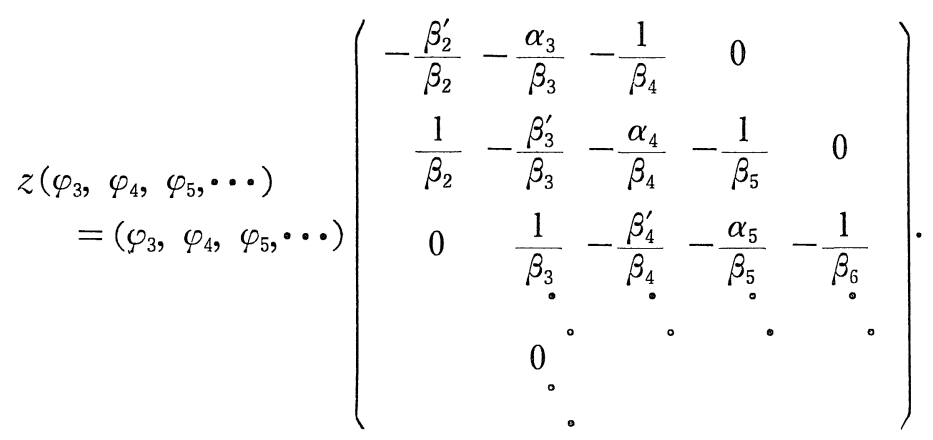

The matrix in the right hand side will be denoted by $L$. Then the pair $\left(\beta_{n+1} \tilde{p}_{n}^{\prime}, \beta_{n+1} \tilde{p}_{n}^{\prime \prime}\right)$ satisfy the adjoint difference equation:

$$
z\left[\begin{array}{cc}
\beta_{2} \tilde{p}_{1}^{\prime} & \beta_{2} \tilde{p}_{1}^{\prime \prime} \\
\beta_{3} \tilde{p}_{2}^{\prime} & \beta_{3} \tilde{p}_{2}^{\prime \prime} \\
\vdots & \vdots
\end{array}\right]=L\left[\begin{array}{cc}
\beta_{2} \tilde{p}_{1}^{\prime} & \beta_{2} \tilde{p}_{1}^{\prime \prime} \\
\beta_{3} \tilde{p}_{2}^{\prime} & \beta_{3} \tilde{p}_{2}^{\prime \prime} \\
\vdots & \vdots
\end{array}\right]
$$

Let $E$ be the Banach space consisting of continuous functions on $\Gamma$ which can be approximated uniformly by polynomials. Then the operator $L$ defines a bounded operator on $E$. We denote by $E^{*}$ the dual space of $E$. Then $e_{n-3}=p_{n}(\zeta)=p_{n}(L) e_{0}, n \geqq 3$, or $e_{n-1}^{*}=\left(\beta_{n+1} \tilde{p}_{n}^{\prime}\right.$, $\left.\beta_{n+1} \tilde{p}_{n}^{\prime \prime}\right)=\beta_{n+1} \tilde{p}_{n}^{\prime}(L) e_{0}^{*}+\beta_{n+1} \tilde{p}_{n}^{\prime \prime}(L) e_{1}^{*}, n \geqq 1$, give dual bases each other of $E$ or $E^{*}$ :

$$
\int_{\Gamma} p_{n+2}(\zeta)\left(\tilde{p}_{m}^{\prime}(\zeta) \mu_{0}(d \zeta)+\tilde{p}_{m}^{\prime \prime}(\zeta) \mu_{1}(d \zeta)\right)=0
$$

for $n \neq m, n, m \geqq 1$. We can also prove the following formulae:

Proposition 4. For $n \geqq 1$,

$$
\tilde{p}_{n}(z)=\int_{\Gamma} \frac{\tilde{p}_{n}^{\prime}(z)-\tilde{p}_{n}^{\prime}(\zeta)}{z-\zeta} \mu_{0}(d \zeta)+\int_{\Gamma} \frac{\tilde{p}_{n}^{\prime \prime}(z)-\tilde{p}_{n}^{\prime \prime}(\zeta)}{z-\zeta} \mu_{1}(d \zeta)
$$

and

$$
u_{n}(z) / u_{0}(z)=\int_{\Gamma} \frac{\tilde{p}_{n}^{\prime}(\zeta) \mu_{0}(d \zeta)}{z-\zeta}+\int_{\Gamma} \frac{\tilde{p}_{n}^{\prime \prime}(\zeta) \mu_{1}(d \zeta)}{z-\zeta}
$$

These imply that the matrix elements of the resolvent of $L,\left(e_{m}^{*}\right.$, $\left.(z-L)^{-1} e_{n}\right)$ are described by the integral representation

$$
\left(e_{m}^{*},(z-L)^{-1} e_{n}\right)=\int_{\Gamma} \frac{p_{n+3}(\zeta)\left(\tilde{p}_{m+1}^{\prime}(\zeta) \mu_{0}(d \zeta)+\tilde{p}_{m+1}^{\prime \prime}(\zeta) \mu_{1}(d \zeta)\right)}{z-\zeta},
$$

$m, n \geqq 0$. In particular, 


$$
\left\{\begin{array}{l}
\omega_{0}=\left(e_{0}^{*},(z-L)^{-1} e_{0}\right), \\
\omega_{1}=\left(e_{1}^{*},(z-L)^{-1} e_{0}\right) .
\end{array}\right.
$$

The operator $L$ has thus been reconstructed from the spectral densities $\mu_{0}(d \zeta), \mu_{1}(d \zeta)$ such that $L$ coincides with the multiplication of the variable $z$ with respect to the bi-orthogonal system of polynomials $p_{n+3}(z)$ and $\left(\tilde{p}_{n+1}^{\prime}(z), \tilde{p}_{n+1}^{\prime \prime}(z)\right)$. This result can be summarized in the following way.

Theorem 1. Under the regularity condition $(\mathscr{H} .1)$, we can uniquely construct by Jacobi-Perron algorithm a bounded operator $L$ on the Banach space $E$ such that its spectral densities coincide with the pair $\mu_{0}(d \zeta)$, $\mu_{1}(d \zeta)$ on $\Gamma$.

\section{§2. Spectral Densities and Lax Type Equations}

We want to show that Lax type equations for the operators constructed in $\S 1$ are equivalent to linear differential equations for their spectral densities.

Let $\omega=\left\{\omega_{\sigma \tau}(\zeta, d \zeta)\right\}_{0 \leqq o \leqq N-1,0 \leqq r \leq M-1}, \zeta \in \boldsymbol{C}$, be a matrix valued continuous 1 -form in a neighbourhood of a rectifiable curve $\Gamma$ in $\boldsymbol{C}$. Let $e_{i}=\left\{e_{i}^{(\tau)}(\zeta), 0 \leqq \tau \leqq M-1\right\}, 0 \leqq i<\infty$, and $e_{j}^{*}=\left\{e_{j}^{*(o)}(\zeta), 0 \leqq \sigma \leqq N-1\right\}$, $0 \leqq j<\infty$, be two linearly independent systems of continuous functions on $\Gamma$ such that the following bi-orthogonal relations hold:

$$
\begin{aligned}
\delta_{i j} & =\left\langle e_{j}^{*}|\omega| e_{i}\right\rangle \\
& =\sum_{\sigma . \tau} \int_{\Gamma} e_{j}^{*(\sigma)}(\zeta) \omega_{\sigma \tau}(\zeta, d \zeta) e_{i}^{(\tau)}(\zeta) .
\end{aligned}
$$

We assume further that there exist two suitable Banach spaces $E$ and $E^{*}$ which are generated by the dual bases $\left\{e_{i}=\left\langle e_{i}^{(\tau)}\right\rangle, 0 \leqq i<\infty\right\}$ and $\left\{e_{i}^{*}=\left\langle e_{i}^{*(\sigma)}\right\rangle, 0 \leqq i<\infty\right\}$ respectively. The duality is denoted by

$$
\begin{aligned}
& E^{*} \times E \mapsto C \\
& \left(x^{*}, y\right) \rightarrow\left(x^{*}, y\right)_{\omega}=\left\langle x^{*}|\omega| y\right\rangle,
\end{aligned}
$$

such that the right hand side of $(2.1)$ is equal to $\left\langle e_{j}^{*}|\omega| e_{i}\right\rangle$. We assume further that $e_{i}, e_{j}^{*}$ and $\omega$ depend differentiably on time $t$ in a neighbourhood $U$ of the origin and satisfy the following order preserving properties: 
$(\mathscr{H} .2) \quad \dot{e}_{i}$ and $\dot{e}_{j}^{*}$ are linear combinations of $e_{i^{\prime}}, i \geqq i^{\prime}$, and $e_{j^{\prime}}^{*}, j \geqq j^{\prime}$, respectively.

We consider linear differential equation for $\omega(\zeta, d \zeta)$ of the following type :

$\left(\mathscr{E}_{1}\right) \quad \dot{\omega}(\zeta, d \zeta)\left(=\frac{d \omega}{d t}(\zeta, d \zeta)\right)=\omega(\zeta, d \zeta) f(t, \zeta)=f^{*}(t, \zeta) \omega(\zeta, d \zeta)$

for suitable continuous square matrices $f(t, \zeta)=\left(f_{\alpha \beta}\right)$ and $f^{*}(t, \zeta)=$ $\left(f_{\alpha \beta}^{*}\right)$ on $\Gamma$ of order $M$ and $N$ respectively. Let $L$ be the operator on $E$ such that its spectral density matrix coincides with $\omega(\zeta, d \zeta)$ :

$$
\left(e_{j}^{*}, L e_{i}\right)_{\omega}=\left\langle e_{j}^{*}|\omega L| e_{i}\right\rangle=\sum_{\sigma, \tau} \int_{\Gamma} \zeta e_{j}^{*(\sigma)}(\zeta) \omega_{\sigma \tau}(\zeta, d \zeta) e_{i}^{(\tau)}(\zeta)
$$

Further we define the operators $f(t, L)$ and $f^{*}\left(t, L^{*}\right)$ respectively as follows :

$$
\begin{aligned}
& \left(e_{j}^{*}, f(t, L) e_{i}\right)_{\omega}=\sum_{\sigma, \rho, \tau} \int_{\Gamma} e_{j}^{*(\sigma)}(\zeta) \omega_{\sigma \rho}(\zeta, d \zeta) f_{\rho \tau}(t, \zeta) e_{i}^{(\tau)}(\zeta) \\
& \left(f^{*}\left(t, L^{*}\right) e_{j}^{*}, e_{i}\right)_{\omega}=\sum_{\sigma \rho, \tau} \int_{\Gamma} e_{j}^{*(\sigma)}(\zeta) f_{\sigma \rho}^{*}(t, \zeta) \omega_{\rho \tau}(\zeta, d \zeta) e_{i}^{(\tau)}(\zeta)
\end{aligned}
$$

Definition. Let $A$ (or $\left.A^{*}\right)$ be a bounded operator on $E$ (or $\left.E^{*}\right)$ defined by an infinite matrix $\left(a_{i j}\right)_{0 \leqq i} j_{j \infty \infty}$ with respect to the bases $\left\{e_{i}\right\}$ and $\left\{e_{j}^{*}\right\}$ such that $\left(e_{j}^{*}, A e_{i}\right)=a_{i j}$. Then $A^{(+)}, A^{(-)}$and $A^{(0)}$ are defined as follows :

$$
\begin{aligned}
& \left(e_{j}^{*}, A^{(+)} e_{\imath}\right)=\left\{\begin{array}{lll}
a_{i j} & \text { if } i>j \\
\frac{1}{2} a_{i i} & \text { if } i=j \\
0 & \text { if } i<j
\end{array}\right. \\
& \left(e_{j}^{*}, A^{(0)} e_{\imath}\right)=\left\{\begin{array}{lll}
0 & \text { if } i \neq j \\
\frac{1}{2} a_{i \imath} & \text { if } i=j
\end{array}\right.
\end{aligned}
$$

and $A^{(-)}=A-A^{(+)}$. Remark $\left(A^{(+)}\right)^{*}=\left(A^{*}\right)^{(-)},\left(A^{(-)}\right)^{*}=\left(A^{*}\right)^{(+)}$.

Then we have the following theorem:

Theorem 2. Suppose that the measures $\omega_{\sigma \tau}(\zeta, d \zeta)$ are non-degenerate and normalized such that we can choose $e_{i}(\zeta), e_{j}^{*}(\zeta)$ as follows: 


$$
\left\{\begin{aligned}
e_{\imath}(\zeta)={ }^{t}(0, \cdots, & 0,1,0, \cdots, 0), \quad 0 \leqq i \leqq M-1 \\
& (1+i)-t h \\
e_{j}^{*}(\zeta)=(0, \cdots, & 0,1,0, \cdots, 0), \quad 0 \leqq j \leqq N-1 \\
& (1+j)-t h
\end{aligned}\right.
$$

Then the differential equation $\left(\mathscr{E}_{1}\right)$ is equivalent to the Lax type nonlinear equations :

$$
\begin{aligned}
\dot{L} & =\left[f(t, L)^{(+)}, L\right], \\
\dot{L}^{*} & =\left[f^{*}\left(t, L^{*}\right)^{(+)}, L^{*}\right] .
\end{aligned}
$$

Proof. We firstly show that $\left(\mathscr{E}_{1}\right)$ implies $\left(\mathscr{E}_{2}\right)$ and $\left(\mathscr{E}_{2}^{*}\right)$. By Leibniz rule, the differentiation of (2.1) with respect to $t$ gives

$$
0=\left\langle\dot{e}_{j}^{*}|\omega| e_{i}\right\rangle+\left\langle e_{j}^{*}|\dot{\omega}| e_{i}\right\rangle+\left\langle e_{j}^{*}|\omega| \dot{e}_{i}\right\rangle .
$$

According to $(\mathscr{H} .2), \dot{e}_{i}$ and $\dot{e}_{j}^{*}$ are described as follows:

$$
\begin{aligned}
& \dot{e}_{i}=\sum_{i \geqq i^{\prime}} \xi_{i^{\prime} i} e_{i^{\prime}}, \\
& \dot{e}_{j}^{*}=\sum_{j \geqq j^{\prime}} \eta_{j^{\prime} j} e_{j^{\prime}}^{*} .
\end{aligned}
$$

Therefore from (2.1), (2.10) and (2.11)

$$
\begin{array}{cc}
\left(e_{j}^{*}, f(t, L) e_{i}\right)_{\omega}+\xi_{j i}=0, & i>j, \\
\left(f^{*}\left(t, L^{*}\right) e_{j}^{*}, e_{i}\right)_{\omega}+\eta_{i j}=0, & i<j,
\end{array}
$$

and

$$
\left(e_{j}^{*}, f(t, L) e_{i}\right)_{\omega}+\xi_{i i}+\eta_{i i}=0, \quad i=j .
$$

By taking $\xi_{i i}=\eta_{i i}$, we can determine uniquely $\xi_{i i}$ as follows:

$$
\xi_{i i}=\eta_{i i}=-\frac{1}{2}\left(e_{i}^{*}, f(t, L) e_{i}\right)_{\omega} .
$$

On the other hand

$$
\begin{aligned}
& \overparen{\left(e_{j}^{*}, L e_{i}\right)_{\omega}}=\frac{\cdot}{\left\langle e_{j}^{*}|\omega L| e_{i}\right\rangle} \\
& =\left\langle\dot{e}_{j}^{*}|\omega L| e_{i}\right\rangle+\left\langle e_{j}^{*}|\dot{\omega} L| e_{i}\right\rangle+\left\langle e_{j}^{*}|\omega L| \dot{e}_{i}\right\rangle \\
& =\sum_{j \geqq j^{\prime}} \eta_{j^{\prime} j}\left\langle e_{j^{\prime}}^{*}|\omega L| e_{i}\right\rangle+\left\langle e_{j}^{*}|\omega f(t, L) L| e_{i}\right\rangle+\sum_{i \geqq i^{\prime}}\left\langle e_{j}^{*}|\omega L| e_{\imath^{\prime}}\right\rangle \xi_{i^{\prime} i} \\
& =-\sum_{j>j^{\prime} \geqq 0}\left\langle e_{j}^{*}|\omega f(t, L)| e_{j^{\prime}}\right\rangle\left\langle e_{j^{\prime}}^{*}|\omega L| e_{i}\right\rangle+\left\langle e_{j}^{*}|\omega f(t, L) L| e_{i}\right\rangle \\
& -\sum_{i>i^{\prime} \geqq 0}\left\langle e_{j}^{*}|\omega L| e_{i^{\prime}}\right\rangle\left\langle e_{i^{\prime}}^{*}|\omega f(t, L)| e_{i}\right\rangle-\frac{1}{2}\left\langle e_{j}^{*}|\omega f(t, L)| e_{j}\right\rangle . \\
& \cdot\left\langle e_{j}^{*} \mid \omega L_{i}^{\prime} e_{i}\right\rangle-\frac{1}{2}\left\langle e_{j}^{*}|\omega L| e_{i}\right\rangle\left\langle e_{i}^{*}|\omega f(t, L)| e_{i}\right\rangle \text {. }
\end{aligned}
$$


By using the relations of completeness, we have

$$
\left\langle e_{j}^{*}|\omega f(t, L) L| e_{i}\right\rangle=\sum_{k=0}^{\infty}\left\langle e_{j}^{*}|\omega f(t, L)| e_{k}\right\rangle\left\langle e_{k}^{*}|\omega L| e_{i}\right\rangle .
$$

Therefore

$$
\begin{aligned}
& \widetilde{\cdot} \overline{\left(e_{j}^{*}, L e_{i}\right)_{\omega}}=\sum_{j^{\prime}>j}\left\langle e_{j}^{*}|\omega f(t, L)| e_{j^{\prime}}\right\rangle\left\langle e_{j^{\prime}}^{*}|\omega L| e_{i}\right\rangle \\
& -\sum_{i>i^{\prime} \geq 0}\left\langle e_{j}^{*}|\omega L| e_{i^{\prime}}\right\rangle\left\langle e_{i^{\prime}}^{*}|\omega f(t, L)| e_{i}\right\rangle+\frac{1}{2}\left\langle e_{j}^{*}|\omega f(t, L)| e_{j}\right\rangle \\
& \cdot\left\langle e_{j}^{*}|\omega L| e_{i}\right\rangle \\
& -\frac{1}{2}\left\langle e_{j}^{*}|\omega L| e_{i}\right\rangle\left\langle e_{i}^{*}|\omega f(t, L)| e_{\imath}\right\rangle \\
& =\sum_{k=0}^{\infty}\left(e_{j}^{*}, f(t, L)^{(+)} e_{k}\right)_{\omega}\left(e_{k}^{*}, L e_{i}\right)_{\omega}-\sum_{k=0}^{\infty}\left(e_{j}^{*}, L e_{k}\right)_{\omega} \\
& =\left(e_{j}^{*},\left[f(t, L)^{(+)}, L\right] e_{i}\right)_{\omega} . \\
& \cdot\left(e_{k}^{*}, f(t, L)^{(+)} e_{\imath}\right)_{\omega}
\end{aligned}
$$

$\left(\mathscr{E}_{2}^{*}\right)$ can be deduced similarly.

Conversely, under the condition (H्.2), suppose that $\left(\mathscr{E}_{2}\right)$ or $\left(\mathscr{E}_{2}^{*}\right)$ holds. Then for $0 \leqq i \leqq N-1$, and $0 \leqq j \leqq M-1$,

$$
\int_{\Gamma} \frac{\omega_{i j}(\zeta, d \zeta)}{z-\zeta}=\left\langle e_{j}^{*}\left|\omega(z-L)^{-1}\right| e_{i}\right\rangle=\left(e_{j}^{*},(z-L)^{-1} e_{\imath}\right)_{\omega} .
$$

By differentiation with respect to $t$, the left hand side is equal to

$$
\int_{\Gamma} \frac{\dot{\omega}_{i j}(\zeta, d \zeta)}{z-\zeta}
$$

while the right hand side is equal to

$$
\begin{aligned}
& \left(e_{j}^{*},\left\{f(t, L)^{(+)}(z-L)^{-1}-(z-L)^{-1} f(t, L)^{(+)}\right\} e_{i}\right)_{\omega} \\
& =\left(e_{j}^{*},\left(f(t, L)-f(t, L)^{(-)}\right)(z-L)^{-1} e_{i}\right)_{\omega} \\
& \quad-\left(e_{j}^{*},(z-L)^{-1} f(t, L)^{(+)} e_{\imath}\right)_{\omega} \\
& =\left(e_{j}^{*}, f(t, L)(z-L)^{-1} e_{i}\right)_{\omega}-\sum_{k=0}^{\infty}\left(f^{*}\left(t, L^{*}\right)^{(+)} e_{j}^{*}, e_{k}\right)_{\omega} \\
& \quad \cdot\left(e_{k}^{*},(z-L)^{-1} e_{i}\right)_{\omega}-\sum_{k=0}^{\infty}\left(e_{j}^{*},(z-L)^{-1} e_{k}\right)_{\omega} \cdot\left(e_{k}^{*}, f(t, L)^{(+)} e_{\imath}\right)_{\omega \cdot}
\end{aligned}
$$

From the normalization $(2.8),\left(f^{*}\left(t, L^{*}\right)^{(+)} e_{j}^{*}, e_{k}\right)_{\omega}$ and $\left(e_{k}^{*}, f(t, L)^{(+)}\right.$ - $\left.e_{i}\right)_{\omega}$ vanish for $0 \leqq i \leqq M-1,0 \leqq j \leqq N-1,0 \leqq k<+\infty$. Therefore (2. 21) is equal to

$$
\left(e_{j}^{*}, f(t, L)(z-L)^{-1} e_{i}\right)_{\omega} .
$$

And we have the equality; 


$$
\int_{\Gamma} \frac{\dot{\omega}_{i j}(\zeta, d \zeta)}{z-\zeta}=\int_{\Gamma} \frac{\{\omega(\zeta, d \zeta) f(t, \zeta)\}_{i j}}{z-\zeta},
$$

which implies $\left(\mathscr{E}_{1}\right)$ :

$$
\dot{\omega}_{i j}(\zeta, d \zeta)=\sum_{k=0}^{M-1} \omega_{i k}(\zeta, d \zeta) f_{k j}(t, \zeta) \text { on } \Gamma .
$$

The theorem has been completely proved.

\section{§ 3. Examples}

(1) Pochhammer integrals (see [3]).

Let $\alpha, \beta, \gamma$ be complex numbers such that $\operatorname{Re} \alpha, \operatorname{Re} \beta, \operatorname{Re} \gamma>0$. The 1 -form $\omega(x, d x)=x^{\alpha}|1-x|^{\beta}|t-x|^{\gamma} d x, t>1$, gives Radon measures $\mu_{0}(d x)$ and $\mu_{1}(d x)$ on each interval $[0,1]$ or $[1, t]$. We define the polynomials $p_{2 k+1}$ of degree $2 k-2, k \geqq 1$, and $p_{2 k}$ of degree $2 k-3$, $k \geqq 2$, respectively by the following formulae. For some constants $c_{k}$, $c_{k}^{\prime}$ and $c_{k}^{\prime \prime}$

$$
\begin{aligned}
c_{k}\left(\frac{d}{d x}\right)^{k-1} & {\left[x^{\alpha+k-1}(x-1)^{\beta+k-1}(x-t)^{\gamma+k-1}\right] } \\
& =(k-1) ! x^{\alpha}(x-1)^{\beta}(x-t)^{\gamma} p_{2 k+1}(x),
\end{aligned}
$$

and

$$
\begin{aligned}
c_{k}^{\prime}\left(\frac{d}{d x}\right)^{k-2} & {\left[x^{\alpha+k-2}(x-1)^{\beta+k-1}(x-t)^{\gamma+k-2}\right] } \\
& +c_{k}^{\prime \prime}\left(\frac{d}{d x}\right)^{k-2}\left[x^{\alpha+k-2}(x-1)^{\beta+k-2}(x-t)^{\gamma+k-1}\right] \\
& =(k-2) ! x^{\alpha}(x-1)^{\beta}(x-t)^{\gamma} p_{2 k}(x),
\end{aligned}
$$

where $c_{k}^{\prime}$ and $c_{k}^{\prime \prime}$ are to be chosen such that

$$
\begin{aligned}
& c_{k}^{\prime} \int_{0}^{1} x^{\alpha+k-2}(x-1)^{\beta+k-1}(x-t)^{\gamma+k-2} d x \\
& \quad+c_{k}^{\prime \prime} \int_{0}^{1} x^{\alpha+k-2}(x-1)^{\beta+k-2}(x-t)^{\gamma+k-1} d x=0 .
\end{aligned}
$$

We express $c_{k}^{\prime}$ and $c_{k}^{\prime \prime}$ by

$$
c_{k}^{\prime}=\rho_{k} \hat{c}_{k}^{\prime}, c_{k}^{\prime \prime}=\rho_{k} \hat{c}_{k}^{\prime \prime},
$$

where

$$
\left\{\begin{array}{l}
\hat{c}_{k}^{\prime}=\int_{0}^{1} x^{\alpha+k-2}(x-1)^{\beta+k-2}(x-t)^{\gamma+k-1} d x, \\
\hat{c}_{k}^{\prime \prime}=-\int_{0}^{1} x^{\alpha+k-2}(x-1)^{\beta+k-1}(x-t)^{\gamma+k-2} d x,
\end{array}\right.
$$


for a suitable constant $\rho_{k}$ which will be determined later.

By partial integration we have the bi-orthogonal relations (1.43) $\sim(1.46)$. We want to compute explicitely $\alpha_{n+1}, \beta_{n+1}$ and $\beta_{n+1}^{\prime}$ in (1.47). We put

$$
\lambda_{0}=-k, \lambda_{1}=\alpha+k-1, \lambda_{2}=\beta+k-1, \lambda_{3}=\gamma+k-1,
$$

and consider the integral of Pochhammer

$$
F\left(\lambda_{0}, \lambda_{1}, \lambda_{2}, \lambda_{3}\right)=\oint(y-x)^{\lambda_{0}} y^{\lambda_{1}}(y-1)^{\lambda_{2}}(y-t)^{\lambda_{3}} d y .
$$

Then $x^{\alpha}(x-1)^{\beta}(x-t)^{\gamma} p_{n}(x)$ can be identified with $F$, except for constant factors :

$$
\begin{aligned}
x^{\alpha}(x-1)^{\beta}(x-t)^{r} p_{2 k+1}(x)= & c_{k} F\left(\lambda_{0}, \lambda_{1}, \lambda_{2}, \lambda_{3}\right), \\
x^{\alpha}(x-1)^{\beta}(x-t)^{\gamma} p_{2 k}(x)= & c_{k}^{\prime} F\left(\lambda_{0}+1, \lambda_{1}-1, \lambda_{2}, \lambda_{3}-1\right) \\
& +c_{k}^{\prime \prime} F\left(\lambda_{0}+1, \lambda_{1}-1, \lambda_{2}-1, \lambda_{3}\right),
\end{aligned}
$$

if the integration (3.7) is taken to be the residue around $x$. A general result about difference systems in [1] shows that $F\left(\lambda_{0}, \lambda_{1}, \lambda_{2}\right.$, $\lambda_{3}$ ) satisfies a maximal overdetermined linear difference equations with coefficients of rational functions of $\lambda_{0}, \lambda_{1}, \lambda_{2}, \lambda_{3}$. In fact this follows from the following lemma.

Lemma 3.1. (Stokes formula). The integral

$$
\begin{gathered}
\int(y-x)^{\lambda_{0}} y^{\lambda_{1}}(y-1)^{\lambda_{2}}(y-t)^{\lambda_{3}}\left\{\frac{d \psi(y)}{d y}+\left(\frac{\lambda_{0}}{y-x}+\frac{\lambda_{1}}{y}\right.\right. \\
\left.\left.+\frac{\lambda_{2}}{y-1}+\frac{\lambda_{3}}{y-t}\right) \psi(y)\right\} d y
\end{gathered}
$$

vanishes for an arbitrary polynomial $\psi(y)$.

We use (3.10) to get an explicit expression of (1.47). $\alpha_{n+1}, \beta_{n+1}$ and $\beta_{n+1}^{\prime}$ in (1.47) can be obtained by solving the following equations:

$$
\begin{array}{r}
-\frac{c_{k+1} y(y-1)(y-t)}{y-x}+\left\{c_{k}^{\prime} \frac{y-x}{y(y-t)}+c_{k}^{\prime \prime} \frac{y-x}{y(y-1)}\right\} \\
+\alpha_{2 k+1} c_{k}+\left(\beta_{2 k+1} x+\beta_{2 k+1}^{\prime}\right)\left\{c_{k+1}^{\prime}(y-1)+c_{k+1}^{\prime \prime}(y-t)\right\} \\
=\frac{d \psi_{1}(y)}{d y}+\left\{\frac{\lambda_{0}}{y-x}+\frac{\lambda_{1}}{y}+\frac{\lambda_{2}}{y-1}+\frac{\lambda_{3}}{y-t}\right\} \psi_{1}(y),
\end{array}
$$

for a polynomial $\psi_{1}(y)$ of degree 3 in case $n=2 k, k \geqq 2$, and 


$$
\begin{aligned}
& -\frac{c_{k+2}^{\prime} y(y-1)^{2}(y-t)+c_{k+2}^{\prime \prime} y(y-1)(y-t)^{2}}{y-x} \\
& +c_{k}+\alpha_{2 k+2}\left\{c_{k+1}^{\prime}(y-1)+c_{k+1}^{\prime \prime}(y-t)\right\} \\
& +\left(\beta_{2 k+2} x+\beta_{2 k+2}^{\prime}\right) c_{k+1} \frac{y(y-1)(y-t)}{y-x} \\
& =\frac{d \psi_{2}(y)}{d y}+\left\{\frac{\lambda_{0}}{y-x}+\frac{\lambda_{1}}{y}+\frac{\lambda_{2}}{y-1}+\frac{\lambda_{3}}{y-t}\right\} \psi_{2}(y),
\end{aligned}
$$

for a polynomial $\phi_{2}(y)$ of degree 4 , in case of $n=2 k+1, k \geqq 1$.

We can solve (3.11) and (3.12) by elementary computation and have the following result:

Lemma 3.2. (i) Case $n=2 k, k \geqq 2$.

$$
\begin{aligned}
& \phi_{1}(y)=-\frac{c_{k+1}}{\lambda_{0}} y(y-1)(y-t)-\frac{\left(c_{k}^{\prime}+c_{k}^{\prime \prime} t\right)}{\lambda_{1} t^{2}}(y-x)(y-1)(y-t) \\
& +\frac{c_{k}^{\prime \prime}}{\lambda_{2}(1-t)}(y-x) y(y-t)+\frac{c_{k}^{\prime}}{\lambda_{3} t^{2}(t-1)}(y-x) y(y-1),
\end{aligned}
$$

and

$$
\begin{aligned}
\frac{\left(3+\lambda_{1}+\lambda_{2}+\lambda_{3}\right)}{\lambda_{0}} c_{k+1}= & \left(3+\lambda_{\infty}\right)\left\{-\frac{c_{k}^{\prime}+c_{k}^{\prime \prime} t}{\lambda_{1} t^{2}}+\frac{c_{k}^{\prime \prime}}{\lambda_{2}(1-t)}\right. \\
& \left.+\frac{c_{k}^{\prime}}{\lambda_{3} t^{2}(t-1)}\right\},
\end{aligned}
$$

where $\lambda_{\infty}$ denotes $\lambda_{0}+\lambda_{1}+\lambda_{2}+\lambda_{3}$.

(ii) Case $n=2 k+1, k \geqq 1$.

$$
\phi_{2}(y)=-\frac{\left(c_{k+2}^{\prime}+c_{k+2}^{\prime \prime}\right) y(y-1)(y-t)}{4+\lambda_{\infty}}\left\{y-\frac{\left(1+\lambda_{2}\right)+\left(1+\lambda_{3}\right) t}{3+\lambda_{1}+\lambda_{2}+\lambda_{3}}\right\},
$$

and

$$
\begin{aligned}
(3+ & \left.\lambda_{1}+\lambda_{2}+\lambda_{3}\right)\left(4+\lambda_{\infty}\right)\left(c_{k+1}^{\prime}+c_{k+1}^{\prime \prime}\right) c_{k} \\
= & -(t-1)\left(c_{k+2}^{\prime}+c_{k+2}^{\prime \prime}\right)\left[c_{k+1}^{\prime}\left(1+\lambda_{2}\right)\left\{\left(1+\lambda_{3}\right)(t-1)-\left(1+\lambda_{1}\right)\right\}\right. \\
& \left.+c_{k+1}^{\prime \prime} t\left(1+\lambda_{3}\right)\left\{\left(1+\lambda_{2}\right)(t-1)+\left(1+\lambda_{1}\right) t\right\}\right] .
\end{aligned}
$$

(3.14) and (3.16) immediately imply

\section{Lemma 3.3.}

$$
c_{k+1}=\delta_{k} \rho_{k}, \quad c_{k}=\delta_{k}^{\prime} \rho_{k+2},
$$

where 
(3.18)

$$
\begin{aligned}
\delta_{k} & =\frac{-k(\alpha+\beta+\gamma+2 k)}{(\alpha+\beta+\gamma+3 k)}\left\{-\frac{\hat{c}_{k}^{\prime}+\hat{c}_{k}^{\prime \prime} t}{(\alpha+k-1) t^{2}}-\frac{\hat{c}_{k}^{\prime \prime}}{(\beta+k-1)(t-1)}\right. \\
& \left.+\frac{\hat{c}_{k}^{\prime}}{(\gamma+k-1) t^{2}(t-1)}\right\},
\end{aligned}
$$

and

$$
\begin{aligned}
\delta_{k}^{\prime}= & \frac{-(t-1)\left(\hat{c}_{k+2}^{\prime}+\hat{c}_{k+2}^{\prime \prime}\right)}{(\alpha+\beta+\gamma+3 k)(\alpha+\beta+\gamma+2 k+1)\left(\hat{c}_{k+1}^{\prime}+\hat{c}_{k+1}^{\prime \prime}\right)} \\
& \cdot\left[\hat{c}_{k+1}^{\prime}(\beta+k)\{(\gamma+k)(t-1)-\alpha-k\}\right. \\
& \left.+\hat{c}_{k+1}^{\prime \prime} t(\gamma+k)\{(\beta+k)(t-1)+(\alpha+k) t\}\right] .
\end{aligned}
$$

As a result,

Corollary. For $k \geqq 0$

$$
\left\{\begin{array}{l}
c_{3 k+1}=\frac{\delta_{3 k} \cdots \delta_{3}}{\delta_{3 k-2}^{\prime} \cdots \delta_{1}^{\prime}} c_{1} \\
c_{3 k+2}=\frac{\delta_{3 k+1} \cdots \delta_{4}}{\delta_{3 k-1}^{\prime} \cdots \delta_{2}^{\prime}} c_{2} \\
c_{3 k+3}=\frac{\delta_{3 k+2} \cdots \delta_{5}}{\delta_{3 k}^{\prime} \cdots \delta_{3}^{\prime}} c_{3}
\end{array}\right.
$$

and

$$
\rho_{k+2}=\frac{c_{k+3}}{\delta_{k+2}} .
$$

Lemma 3.4 (Characterization of $\hat{c}_{k}^{\prime}$ and $\left.\hat{c}_{k}^{\prime \prime}\right), \hat{c}_{k}^{\prime}, \hat{c}_{k}^{\prime \prime}$ satisfy the following recurrence relatlon:

$$
\left(\hat{c}_{k+1}^{\prime}, \hat{c}_{k+1}^{\prime \prime}\right)=\left(\hat{c}_{k}^{\prime}, \hat{c}_{k}^{\prime \prime}\right)\left[\begin{array}{ll}
a_{11}(k) & a_{12}(k) \\
a_{21}(k) & a_{22}(k)
\end{array}\right],
$$

where $a_{11}(k), a_{21}(k), a_{12}(k)$ and $a_{22}(k)$, in view of $(3.6)$, denote rational functions of $k$ as follows:

$$
\begin{aligned}
a_{11}(k)= & \frac{\lambda_{1} t(t-1)}{\lambda_{\infty}\left(\lambda_{\infty}+1\right)\left(\lambda_{\infty}+2\right)}\left\{-\lambda_{2}\left(1+\lambda_{2}\right)+\frac{\left(\lambda_{0}+\lambda_{2}\right)\left(\lambda_{0}+\lambda_{2}+1\right)}{t}\right. \\
& \left.+\frac{\lambda_{0}\left(1+\lambda_{1}+\lambda_{2}\right)}{t-1}\right\}, \\
a_{21}(k)= & -\frac{\lambda_{2} t^{2}(t-1)}{\lambda_{\infty}\left(\lambda_{\infty}+1\right)\left(\lambda_{\infty}+2\right)}\left\{\left(\lambda_{0}+\lambda_{1}\right)\left(1+\lambda_{2}\right)-\frac{\lambda_{1}\left(1+\lambda_{0}+\lambda_{2}\right)}{t}\right. \\
& \left.-\frac{\lambda_{0}^{2}}{t-1}\right\},
\end{aligned}
$$




$$
\begin{aligned}
a_{12}(k)=\frac{-\lambda_{1} t(t-1)}{\lambda_{\infty}\left(\lambda_{\infty}+1\right)\left(\lambda_{\infty}+2\right)}\{ & \lambda_{2}\left(1+\lambda_{0}+\lambda_{1}\right)-\frac{\left(\lambda_{0}+\lambda_{2}\right)\left(1+\lambda_{1}\right)}{t} \\
& \left.-\frac{\lambda_{0}^{2}}{t-1}\right\},
\end{aligned}
$$

and

$$
\begin{aligned}
a_{22}(k)=\frac{\lambda_{2} t^{2}(t-1)}{\lambda_{\infty}\left(\lambda_{\infty}+1\right)\left(\lambda_{\infty}+2\right)}\{ & -\left(\lambda_{0}+\lambda_{1}\right)\left(\lambda_{0}+\lambda_{1}+1\right) \\
& \left.-\frac{\lambda_{1}\left(1+\lambda_{1}\right)}{t}+\frac{\lambda_{0}\left(1+\lambda_{1}+\lambda_{2}\right)}{t-1}\right\} .
\end{aligned}
$$

Proof. In view of (3.5), $\hat{c}_{k}^{\prime}$ and $\hat{c}_{k}^{\prime \prime}$ are equal to $F\left(0, \lambda_{1}-1, \lambda_{2}-1\right.$, $\left.\lambda_{3}\right)$ and $-F\left(0, \lambda_{1}-1, \lambda_{2}, \lambda_{3}-1\right)$ respectively. Then the relation (3.22) implies that there exist polynomials $\phi_{1}(x)$ and $\phi_{2}(x)$ of degree 2 such that

$$
\begin{aligned}
x-t= & \frac{a_{11}(k)}{x(x-1)}+\frac{a_{21}(k)}{x(x-t)}+\frac{d \phi_{1}(x)}{d x} \\
& +\left(\frac{\lambda_{1}}{x}+\frac{\lambda_{2}}{x-1}+\frac{\lambda_{3}}{x-t}\right) \psi_{1}(x),
\end{aligned}
$$

and

$$
\begin{aligned}
x-1= & \frac{a_{12}(k)}{x(x-1)}+\frac{a_{22}(k)}{x(x-t)}+\frac{d \psi_{2}(x)}{d x} \\
& +\left(\frac{\lambda_{1}}{x}+\frac{\lambda_{2}}{x-1}+\frac{\lambda_{3}}{x-t}\right) \psi_{2}(x) .
\end{aligned}
$$

$a_{11}(k), a_{21}(k), a_{12}(k)$ and $a_{22}(k)$ can be determined as in Lemma 3.2 by explicit computation of these equations.

Proposition 4. (i) $\alpha_{2 k+1}, \beta_{2 k+1}$ and $\beta_{2 k+1}^{\prime}$ are rational functions of $c_{k}, c_{k+1}, c_{k}^{\prime}, c_{k}^{\prime \prime}, c_{k+1}^{\prime}, c_{k+1}^{\prime \prime}, \alpha, \beta, \gamma, k$ and $t$.

(ii) $\alpha_{2 k+2}, \beta_{2 k+2}$ and $\beta_{2 k+2}^{\prime}$ are rational functions of $c_{k}, c_{k+1}, c_{k+1}^{\prime}$, $c_{k+1}^{\prime \prime}, c_{k+2}^{\prime}, c_{k+2}^{\prime \prime}, \alpha, \beta, \gamma, k$ and $t$. Here $c_{k}, \rho_{k}, \hat{c}_{k}^{\prime}$ and $\hat{c}_{k}^{\prime \prime}$ are obtained by the formulae (3.9), (3.21) and (3.4) respectively and $\hat{c}_{k}^{\prime}$ and $\hat{c}_{k}^{\prime \prime}$ satisfy the recurrence relation (3.22).

The spectrum of $L$ coincides with the intervals $[0, t]$. The supports of its spectral densities $\mu_{0}(d \zeta)$ and $\mu_{1}(d \zeta)$ are equal to $[0,1]$ and $[1, t]$ respectively. $\mu_{0}(d \zeta)$ and $\mu_{1}(d \zeta)$ satisfy a linear differential equation as function of $t$ : 


$$
\left(\dot{\mu}_{0}(d \zeta), \dot{\mu}_{1}(d \zeta)\right)=\left(\mu_{0}(d \zeta), \mu_{1}(d \zeta)\right) \frac{\gamma}{t-\zeta} .
$$

According to Theorem 2 in $\S 2$, we have

\section{Proposition 5.}

$$
\dot{L}=\left[L,\left(\frac{\gamma}{t-L}\right)^{(+)}\right]
$$

\section{(2) Periodic case (See [18]).}

We assume further that the matrix elements of $L$ have period $m$ :

$$
\alpha_{n+m}=\alpha_{n}, \quad \beta_{n+m}=\beta_{n}, \quad \beta_{n+m}^{\prime}=\beta_{n}^{\prime},
$$

for $n \geqq l \geqq 2$. The matrix $L$ can be extended to a periodic matrix $\tilde{L}$ such that (3.11) holds for non-positive integers. Then ${ }^{t}\left(u_{l}, v_{l}, w_{l}\right)$ satisfies the characteristic equation

$$
\left[y-A_{l, m}(z)\right]\left[\begin{array}{c}
u_{l} \\
v_{l} \\
w_{l}
\end{array}\right]=0
$$

where $A_{l, m}$ denotes the product

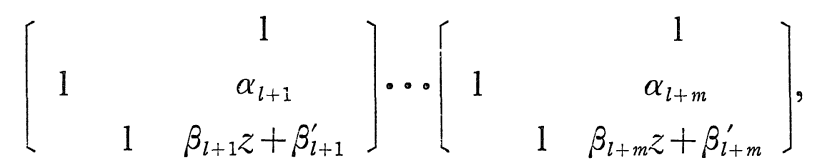

and $y$ is an eigenvalue of $A_{l, m}(z) . \quad y$ satisfies an integral algebraic equation

$$
y^{3}-\left(a_{0}+a_{1} z+\cdots+a_{2 k} z^{2 k}\right) y^{2}+\left(b_{0}+b_{1} z+\cdots+b_{k} z^{k}\right) y-1=0,
$$

or

(3.35) $y^{3}-\left(a_{0}+a_{1} z+\cdots+a_{2 k+1} z^{2 k+1}\right) y^{2}+\left(b_{0}+b_{1} z+\cdots+b_{k} z^{k}\right) y-1=0$, according as $m=2 k$ or $2 k+1$. We denote by $\mathscr{L}_{m}$ the algebraic curve defined by (3.34) or (3.35). Then we can make use of the same method as in [17] and prove the following:

Proposition 6. Under the assumption that

$$
\begin{cases}a_{2 k}\left(b_{k}^{2}-4 a_{2 k}\right) \neq 0 & \text { for } m=2 k, \\ a_{2 k+1} \neq 0 & \text { for } m=2 k+1,\end{cases}
$$


the genus of $\mathscr{L}_{m}$ is equal to $3 k-2$ for $m=2 k$ or $3 k$ for $m=2 k+1$.

i) The divisor of $y$ in $\mathscr{L}_{m}$ is written as follows

$$
\begin{array}{ll}
(y)=-2 k P+k\left(P^{\prime}+P^{\prime \prime}\right) & \text { for } m=2 k, \\
(y)=-(2 k+1) P+(2 k+1) P^{\prime} & \text { for } m=2 k+1,
\end{array}
$$

where $P, P^{\prime}, P^{\prime \prime}(m=2 k)$ or $P, P^{\prime}(m=2 k+1)$ denote effective divisors of degree 1 lying over $z=\infty$ :

or

$$
\begin{aligned}
& (z)+P+P^{\prime}+P^{\prime \prime} \geqq 0, \\
& (z)+P+P^{\prime} \geqq 0 .
\end{aligned}
$$

ii) $u_{n+l}$ and $\left(\frac{v_{n+l}}{u_{n+l}}, \frac{w_{n+l}}{u_{n+l}}\right)$ are meromorphic functions on $\mathscr{L}_{m}$ whose divisors satisfy

$$
\begin{gathered}
\left(u_{2 j+l}\right)=j\left(2 P-P^{\prime}-P^{\prime \prime}\right)+\mathscr{D}_{2 j+l}-\mathscr{D}_{l}, \\
\left\{\begin{array}{l}
\left(\frac{v_{2 j+l}}{u_{2 j+l}}\right)=-P^{\prime}-P^{\prime \prime}+\mathscr{D}_{2 j-1+l}^{\prime}-\mathscr{D}_{2 j+l}, \\
\left(\frac{w_{2 j+l}}{u_{2 j+l}}\right)=-P+\mathscr{D}_{2 j-1+l}-\mathscr{D}_{2 j+l},
\end{array}\right. \\
\left(\begin{array}{l}
\left.u_{2 j+1+l}\right)=(2 j+1) P-j\left(P^{\prime}+P^{\prime \prime}\right)+\mathscr{D}_{2 j+1+l}-\mathscr{D}_{l}, \\
\left(\frac{v_{2 j+1+l}}{u_{2 j+1+l}}\right)=\mathscr{D}_{2 j+1-l}^{\prime}-\mathscr{D}_{2 j+1+l}, \\
\left(\frac{w_{2 j+1+l}}{u_{2 j+1+l}}\right)=-P+P^{\prime}+P^{\prime \prime}+\mathscr{D}_{2 j+l}-\mathscr{D}_{2 j+1+l},
\end{array}\right.
\end{gathered}
$$

in case $m=2 k$, or

$$
\begin{gathered}
\left(u_{n+l}\right)=n\left(P-P^{\prime}\right)+\mathscr{D}_{n+l}-\mathscr{D}_{l}, \\
\left\{\begin{array}{l}
\left(\frac{v_{n+l}}{u_{n+l}}\right)=-P^{\prime}+\mathscr{D}_{n+l}^{\prime}-\mathscr{D}_{n+l}, \\
\left(\frac{w_{n+l}}{u_{n+l}}\right)=P^{\prime}-P+\mathscr{D}_{n-1+l}-\mathscr{D}_{n+l}
\end{array}\right.
\end{gathered}
$$

in case $m=2 k+1$.

\section{Conversely}

Proposition 7. Suppose that an arbitrary sequence of complex numbers $\left\{a_{0}, a_{1}, \cdots, a_{2 k}, b_{0}, \cdots, b_{k}\right\}$ for $m=2 k$ or $\left\{a_{0}, \cdots, a_{2 k+1}, b_{0}, \cdots, b_{k}\right\}$ for $m=2 k+1$ is given such that (3.36) or (3.37) holds. We choose $\left(u_{l}, v_{l}, w_{l}\right)$ for $l \geqq 2$ such that 


$$
\left\{\begin{array}{l}
\left(\frac{v_{l}}{u_{l}}\right)=-P^{\prime}-P^{\prime \prime}+\mathscr{D}_{l-1}^{\prime}-\mathscr{D}_{l}, \\
\left(\frac{w_{l}}{u_{l}}\right)=-P+\mathscr{D}_{l-1}-\mathscr{D}_{l}, \quad m=2 k,
\end{array}\right.
$$

or

$$
\left\{\begin{array}{l}
\left(\frac{v_{l}}{u_{l}}\right)=-P^{\prime}+\mathscr{D}_{l}^{\prime}-\mathscr{D}_{l} \cdot \\
\left(\frac{w_{l}}{u_{l}}\right)=P^{\prime}-P+\mathscr{D}_{l-1}-\mathscr{D}_{l}, \quad m=2 k+1,
\end{array}\right.
$$

where $\mathscr{D}_{l}$ denotes a regular effective divisor on $\mathscr{L}_{m}$. Then we have the periodic Jacobi-Perron algorithm for the pair $\left(v_{l} / u_{l}, w_{l} / u_{l}\right)$ of period $2 k$ or $2 k+1$ such that

$$
\begin{aligned}
& y u_{2 k+l}=u_{l}, \\
& \frac{v_{2 k+l}}{u_{2 k+l}}=\frac{v_{l}}{u_{l}}, \quad \frac{w_{2 k+l}}{u_{2 k+l}}=\frac{w_{l}}{u_{l}},
\end{aligned}
$$

or

$$
\begin{aligned}
& y u_{2 k+1+l}=u_{l}, \\
& \frac{v_{2 k+l+1}}{u_{2 k+l+1}}=\frac{v_{l}}{u_{l}}, \quad \frac{w_{2 k+l+1}}{u_{2 k+l+1}}=\frac{w_{l}}{u_{l}} .
\end{aligned}
$$

In particular we consider the case $m=2$. Then $\mathscr{L}_{2}$ is an algebraic curve defined by the equation where we put $y=h^{-1}$ :

$$
F\left(z, h, h^{-1}\right)=\left|\begin{array}{ll}
-\frac{\beta_{2}^{\prime}}{\beta_{2}}-\frac{h}{\beta_{2}} & \frac{1}{\beta_{3}}-\frac{\alpha_{3}}{\beta_{3}} h \\
-\frac{\alpha_{2}}{\beta_{2}}+\frac{h^{-1}}{\beta_{2}} & -z-\frac{\beta_{3}^{\prime}}{\beta_{3}}-\frac{h}{\beta_{3}}
\end{array}\right|,
$$

and $h, h^{\prime}, h^{\prime \prime}$ be three roots of it. If $|h| \neq 1,\left|h^{\prime}\right| \neq 1,\left|h^{\prime \prime}\right| \neq 1$, we may assume that there are only two cases, (i) $|h|<1,\left|h^{\prime}\right|>1,\left|h^{\prime \prime}\right|>1$ or (ii) $|h|<1,\left|h^{\prime}\right|<1,\left|h^{\prime \prime}\right|>1$. For $|z| \gg 1$, case (i) occurs. There exist three linearly independent quasi-periodic solutions for (1.47)

$$
\varphi^{(0)}=\left\{\varphi_{i}^{(0)}\right\}, \varphi^{(1)}=\left\{\varphi_{i}^{(1)}\right\}, \varphi^{(2)}=\left\{\varphi_{i}^{(2)}\right\},
$$

such that

$$
\left\{\begin{array}{l}
\varphi_{n+2}^{(0)}=h \varphi_{n}^{(0)}, \\
\varphi_{n+2}^{(1)}=h^{\prime} \varphi_{n}^{(1)}, \\
\varphi_{n+2}^{(2)}=h^{\prime \prime} \varphi_{n}^{(2)} .
\end{array}\right.
$$


Consequently $\varphi^{(0)} \in l^{2}[0, \infty)$ and none of non-trivial linear combinations of $\varphi^{(1)}$ and $\varphi^{(2)}$ belong to $l^{2}[0, \infty)$. We have

$$
\begin{aligned}
& \varphi_{n}^{(0)}=O\left(z^{-n-1}\right), \\
& \varphi_{n}^{(0)}=\tilde{p}_{n+1}(z)+\varphi_{0}^{(0)} \tilde{p}_{n+1}^{\prime}(z)+\varphi_{1}^{(0)} \tilde{p}_{n+1}^{\prime \prime}(z),
\end{aligned}
$$

for $n \geqq 0$. $\varphi_{n}^{(0)}$ coincides with $u_{n+1}$ in (1.24). Suppose further that the algebraic equation (3.34) defines a Galois covering over $\boldsymbol{C} P$. Then by choosing a suitable variable for $z$ we may assume that $h$ is equal to

$$
h=z+\sqrt[3]{1-z^{3}}
$$

and the matrix $L$ has the following expression

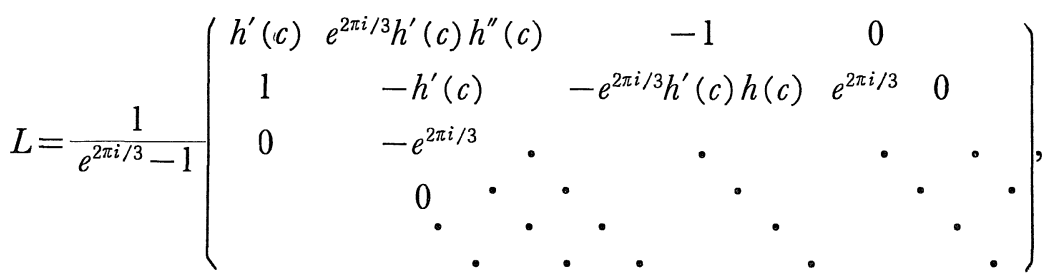

where $c \in \boldsymbol{C}$. We denote by $\Gamma$ the set of all $z \in \boldsymbol{C}$ such that $|h|=1$ or $\left|h^{\prime}\right|=1$ or $\left|h^{\prime \prime}\right|=1$. Then $\Gamma$ is a real algebraic curve having branch points $1, e^{2 \pi i / 3}, e^{4 \pi i / 3}$, and $\boldsymbol{C}-\Gamma$ has four components which we denote by $\mathscr{D}_{1}, \mathscr{D}_{2}, \mathscr{D}_{3}$ and $\mathscr{D}_{4}$ (see the figure).

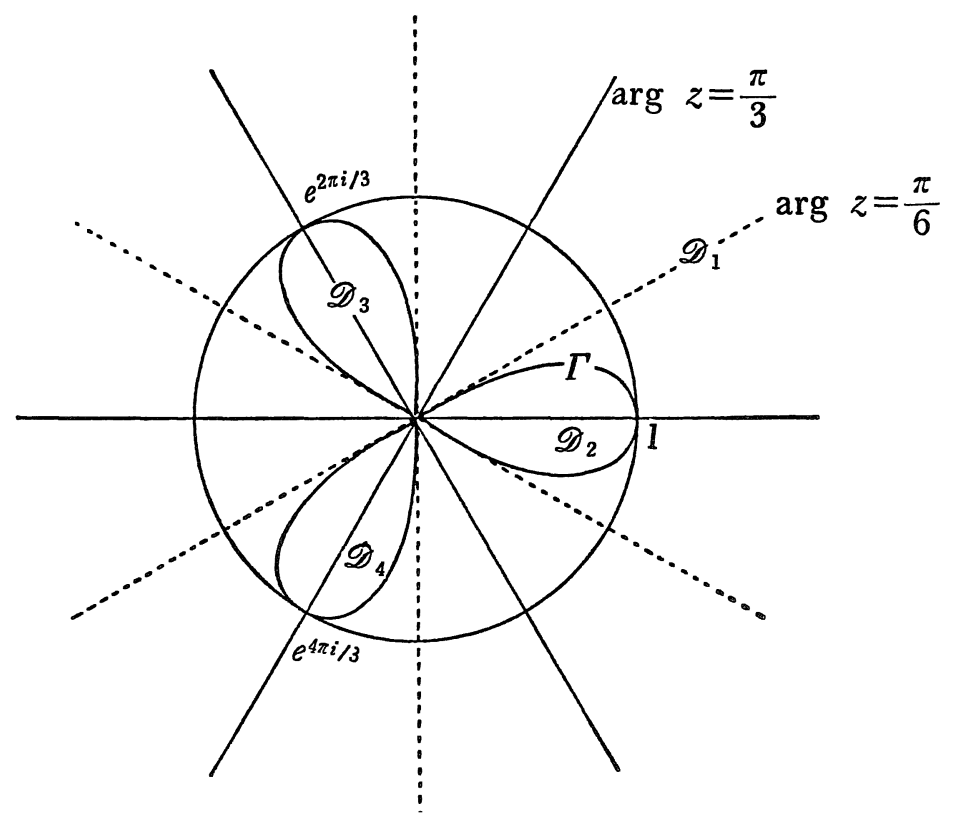


Proposition 8. The spectrum of $L$ consists of $\overline{\mathscr{D}}_{2}, \overline{\mathscr{D}}_{3}$ and $\overline{\mathscr{D}}_{4}$. The interior part of $\boldsymbol{C}-\Gamma, \mathscr{D}_{2} \cup \mathscr{D}_{3} \cup \mathscr{D}_{4}$ coincides with the residual spectrum of $L$.

Proof. We denote by $\left[\begin{array}{lll}0, & 1, & 2 \\ i, & j & k\end{array}\right]$ and $\left[\begin{array}{cc}a, & b \\ i, & j\end{array}\right]$ the determinants

$$
\left|\begin{array}{ccc}
\varphi_{i}^{(0)} & \varphi_{j}^{(0)} & \varphi_{k}^{(0)} \\
\varphi_{i}^{(1)} & \varphi_{j}^{(1)} & \varphi_{k}^{(1)} \\
\varphi_{i}^{(2)} & \varphi_{j}^{(2)} & \varphi_{k}^{(2)}
\end{array}\right|,\left|\begin{array}{cc}
\varphi_{i}^{(a)} & \varphi_{j}^{(a)} \\
\varphi_{i}^{(b)} & \varphi_{j}^{(b)}
\end{array}\right|,
$$

respectively. Then the resolvent $(z-L)^{-1}$ can be described as the Green kernel $G(i, j \mid z), i, j \geqq 2$, as follows :

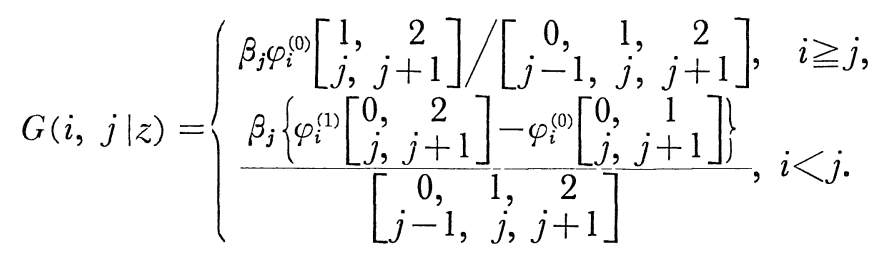

If $z$ lies in the resolvent set of $L$, then $(z-L)^{-1}$ and $\left(z-L^{*}\right)^{-1}$ must be bounded operators, so that

$$
\left\{\begin{array}{l}
\sum_{j=0}^{\infty}\left|G\left(i_{0}, j \mid z\right)\right|^{2}<\infty, \\
\sum_{i=0}^{\infty}\left|G\left(i, j_{0} \mid z\right)\right|^{2}<\infty,
\end{array}\right.
$$

for fixed $i_{0}$ and $j_{0}$. From (3.53), we must have $|h|<1,\left|h^{\prime}\right|>1$ and $\left|h^{\prime \prime}\right|>1$. This implies the proposition.

Remark. In Examples 1, the matrix $L$ has only continuous spectrum while in Example 2, $L$ has residual spectrum. It seems to be interesting to give a criterion whether $L$ of $(1.47)$ has any residual spectrum or not.

The authors are thankful to Professor S. Takenaka for having given them a preliminary numerical computation by computer. This was very helpful to them.

\section{References}

[1] Aomoto, K., Les équations aux différences linéaires et les intégrales des fonctions multi-formes, J. Fac. Sci. Univ. of Tokyo, 22 (1975), 271-297.

[2] Lax equation and the spectral density of Jacobi matrices for orthogonal 
polynomials, preprint 1981.

[3] Appell, P. et Kampé de Fériet, J., Fonctions hypergéométriques et hypersphériques, GauthiersVillars, Paris, 1926.

[4] Berezanski, J. M., Expansions in eigenfunctions of self-adjoint operators, Transl. of Math, 17 (1968).

[5] Bernstein, L., The Jacobi-Perron algorithm, its theory and application, Springer Lec. Notes, 207 (1971).

[6] Case, M. and Kac, M., A discrete version of the inverse scattering problem, J. Math. Phys., 14 (1973), 594-603.

[7] Chudonovsky, G. V., The inverse scattering problem and application to arithmetics, Springer Lec. Notes in Phys. 120 (1980), 155-198.

[8] Flaschka, H., The Toda lattices II, Prog. of Theor. Phys., 15 (1974), 703-716.

[9] Hirota, R., Direct methods in soliton theory, Springer, 17 (1980).

[10] Kac, M. and Van Moerbeke, P., On an explicitely soluble system of nonlinear differential equations related to certain Toda lattices, Adv. in Math., 16 (1975), 160-169.

[11] Kato, Y., On the spectral density of periodic Jacobi matrices, Nonlinear integrable systemsClassical Theory and Quantum Theory, World Sci., Pub., 1983.

[12] Kodaira, K., On ordinary differential equations of any order and the corresponding eigenfunction expansions, Amer. J. Math., 72 (1950), 502-544.

[13] Mahler, K., Zur Approximationen der Exponentialfunktion und des Logarithms, I, II, Crelle J., 166 (1932), 118-136.

[14] Lectures on transcendental numbers, Springer Lec. Notes, 546 (1976).

[15] Mergelyan, S. N., Uniform approximation of functions of complex variables, Uspehi Mat. Nauk, 7 (1952), 31-122.

[16] Mckean, H. P., Bousinesq's equation on the circle, Courant Inst. of Math. Sci., 1980.

[17] Minkowski, H., Über periodische Approximationen algebraischer Zahlen, Acta Math., 26 (1902), 333-351.

[18] Van Moerbeke, P. and Mumford, D., The spectrum of difference operators and algebraic curves, Acta Math., 143 (1979), 93-154.

[19] Stone, M. H., Linear transformations in Hilbert space and their applications to analysis, A. M. S., 1932.

[20] Szegö, G., Orthogonal polynomials, A. M. S., 1938. 